\title{
GEOMETRIC PARAMETERS OF CUTTING TOOLS THAT CAN BE USED FOR FORMING SIDED SURFACES WITH VARIABLE PROFILE
}

\begin{abstract}
This article describes machining technology of polyhedral surfaces with varying profile, which is provided by planetary motion of multiblade block tools. The features of the technology and urgency of the problem is indicated. The purpose of the study is to determine the minimum value of the clearance angle of the tool. Also, the study is carried out about changing the value of the front and rear corners during the formation of polygonal surface using a planetary gear. The scheme of calculating the impact of various factors on the value of the minimum clearance angle of the tool and kinematic front and rear corners of the instrument is provided. The mathematical formula for calculating the minimum clearance angle of the tool is given. Also, given the formula for determining the front and rear corners of the tool during driving. This study can be used in the calculation of the design operations forming multifaceted external surfaces with a variable profile by using the planetary gear.

Keywords: edge surface, polyhedral surface, variable profile, payment verification, the planetary gear
\end{abstract}

\section{Introduction}

In modern engineering there is a widespread usage of different types of details. Type of detail largely determines the manufacturing technology of this type of detail and model manufacturing process. One of the most common types of machinery parts are body rotation parts. They are different for the service, intended constructive shape, size and material. Among the huge variety of parts in this class we must select a group of parts with bodies of revolution having a surface with a cross section other than a circle. These details include prismatic momentum transferring shafts with the surface having the form of a cone. Separate portions of the various parts, such as a spring used in absorbers of railway carriages. Since the development of the technology these components are of particular interest for manufacturing. In this article there are considered and described technological operations of manufacturing end faces of the spring pieces of railway vehicles.

An overview of the methods used for the manufacture of faceted surfaces with variable profile, namely, hot forging, hot rolling, showed that the considered methods are either have low productivity or expensive and have limited application. Thus, they are uneconomic in terms of manufacturing faceted surfaces on automatic machining lines. To implement the method of forming under the automatic line this is necessary to prepare the workpieces, namely turning the workpiece to assure it will have a conical shape in order to reduce stress during the deformation and to ensure the necessary stability of the stamp, which greatly reduces the performance of automatic lines [1].

Thus, the urgent task is to study the design and technological parameters of the process of shaping the verged profiles and development of tooling for the formation of such surfaces on automatic lines.

\section{Fabrication methods for sided surfaces}

For formation of the faceted surfaces using a planetary motion and variable profile along the length of the cross section it is necessary to calculate the planetary gear, namely, to determine the gear ratio selected and processing method. There are the following ways of forming surfaces with planetary motion: the way of an approximate straight line of a polyhedron with hypotrochoid ovals; way to approximate a straight line portion of the elongated polyhedron hypotrocho [2].

To implement the method in the conditions of automatic line it is necessary to do the shaping of sided surface in one setpiece. Ensure that it is possible, through the special design of prefabricated metal cutting tools, in which its cutting edge will be oriented at an angle to the axis of the workpiece surface, and the length corresponds to the length of the formed faces.

Opposite sides of the hexagonal portion of the workpiece has a different slant to its axis. Therefore, forming a single setup, you must have one pair of cutting tool blades with different

\footnotetext{
SOUTHWEST STATE UNIVERSITY, 50 YEARS OF OKTOBER STREET, 94, KURSK, RUSSIAN FEDERATION

* Corresponding author: agrechuhin@mail.ru
} 

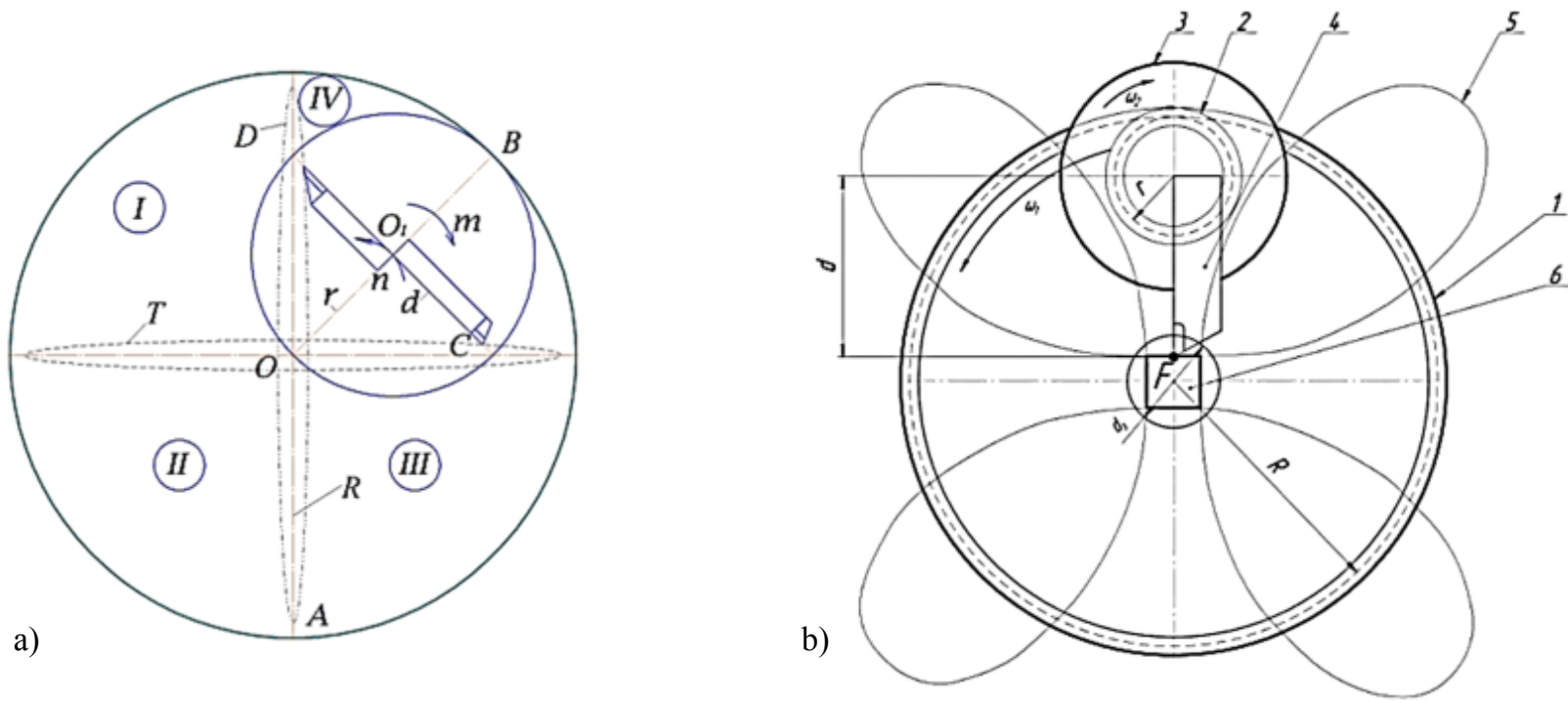

Fig. 1. Scheme shaping sided surfaces with variable profile: a) - the straight line method for approximating polyhedron, where $T, D$ - path cutters, $m, n,-$ rotational movement, $d$ - fly cutting edges, $O, O_{1}$ - centers of gears; b) - a method for approximating a straight line portion of the polyhedron elongated hipotrochoid: 1 - ring gear; 2 - mobile gear; 3 -a tool; 4 -knife; 5 - the trajectory described by the apex of the cutting edge; 6 - detail; $\omega_{1}$ - angular velocity of the moving gear 2 relative to the center of the fixed gear $1 ; \omega_{2}$ - the angular velocity of the moving gears 2 regarding to its own center; $d_{z}$ - workpiece diameter

slopes providing processing of different faces. With this method of processing the workpiece is fixed in a fixing mechanism, processing occurs through the pair of planetary rotation of cutting tool and the workpiece axis.

Thus, to implement the method of forming sided surfaces with variable profile a method of replacing a straight line polyhedron hypotrohoids as ovals was chosen. A s in this case, you can create different departure cutting edges for forming a rectangular profile. The method consists in the fact that one or two of the knife tools, following a closed path, provide the shaping of two opposing edges of the workpiece. The machining of polyhedra with two, four, six, etc. faces tool provided with a suitable amount of knives - one, two, three, etc., have equal gab. Assume $R / r=2$, where $R$ - radius of the planetary gear, $r$ - radius of the satellite [3].

Kinematics of planetary formation has processing characteristics. One feature is the back surface of the contact surface of the tool and workpiece during machining. Consequently, the absence of contact with the rear surface of the tool in the workpiece during processing is the main condition for which performance is necessary to determine the magnitude of the minimum value of the angle adjustable cutting tool [4].

\section{Definition of the minimum allowed value of the side angle}

To determine the minimum acceptable value of tool angle, consider a processing circuit sided surfaces with variable profile tool with planetary motion, which is presented in Figure 3.

Tool, making movement in the planetary gear with internal teeth, rotates on its axis. The trajectories of the movements of the tool and a point on the rear surface of the trochoid represent 2,1 , respectively [5].

Figure 2a shows a diagram of formation, in which the value of the posterior angle of the tool is more than the minimum allowed. In this case, the path of movement of the tool tip point and a rear surface cross-section beyond the workpiece at the point $A$ and the rear surface of the tool does not make contact with the workpiece surface.

Consider the circuit formation shown in Figure 2c, at the time of processing, when the trajectory of the vertex of the cutting edge intersects with the trajectory of the rear surface of the instrument in one of the vertices of the workpiece section, and the trajectory of the rear surface section intersects in two points $A / /, B / /$. In this scheme, the value of adjustable cutting tools is less than its minimum value. Rear contact surface of the tool and the workpiece surface along the curve $A$ will // $B / /$. At the same time, final surface with the given parameters of the cross-section is impossible, since the process will be paired with a punch tool on the workpiece surface.

The task of determining the minimum value of the angle is adjustable in determining the intersection point of the trajectories of the cutting edge and the top point of the rear surface at a point $A$ / figure $2 b$ within a section of the workpiece. The angle formed by the top of the cutting edge, the rear surface of the tool and the workpiece plane in this case constitute the minimum allowable clearance angle of the cutting tool.

To construct an analytical determination according minimum, the clearance angle of the tool during shaping sided surfaces with variable profile by turning planetary, it is necessary to determine the point of intersection of movement trajectories of the cutting edge and the top point of the back surface, namely the trochoid 1 and 2. [3] 


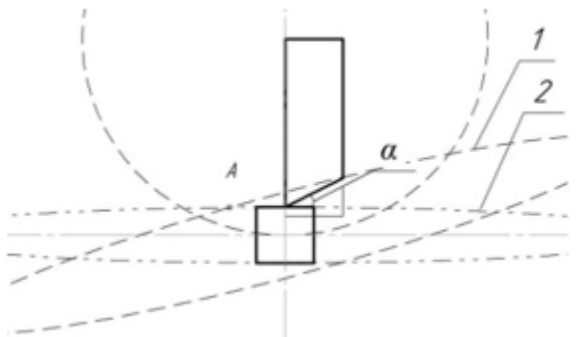

a)

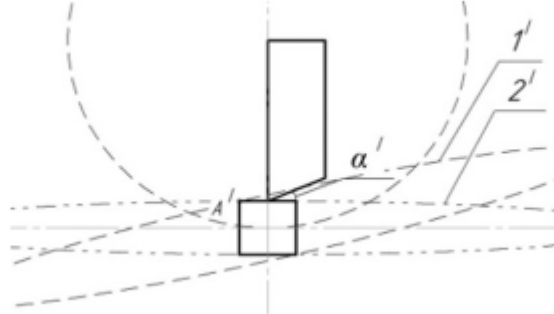

b)

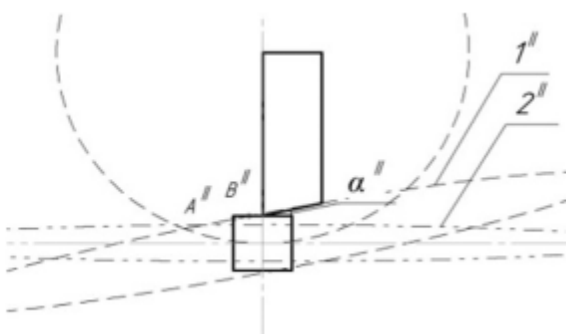

c)

Fig. 2. Scheme of sided shaping surfaces with a variable profile tool with a planetary motion of: a) - the circuit without contact the rear surface of the tool and the workpiece surface - 1 - trajectory of the rear surface 2 - the trajectory of the cutting edge of the vertices, A - the point of intersection of the trajectories of the front and rear surface points; b) - the point of contact with the circuit surface of the rear surface of the tool and the workpiece $-1 /$ - trajectory of the rear surface, $2 /$ - trajectory of the cutting edge of the vertices, the A / - the intersection point of the trajectories of the front and rear surface points of the top section at a point of treatment; $\mathrm{c}$ ) - scheme with rear contact surface of the tool and the workpiece surface through the $-1 / /$ - trajectory of the rear surface, $2 / /$ - trajectory of the cutting edge of the vertices, A // B // - curve intersection point of the trajectories of the front and the rear surface

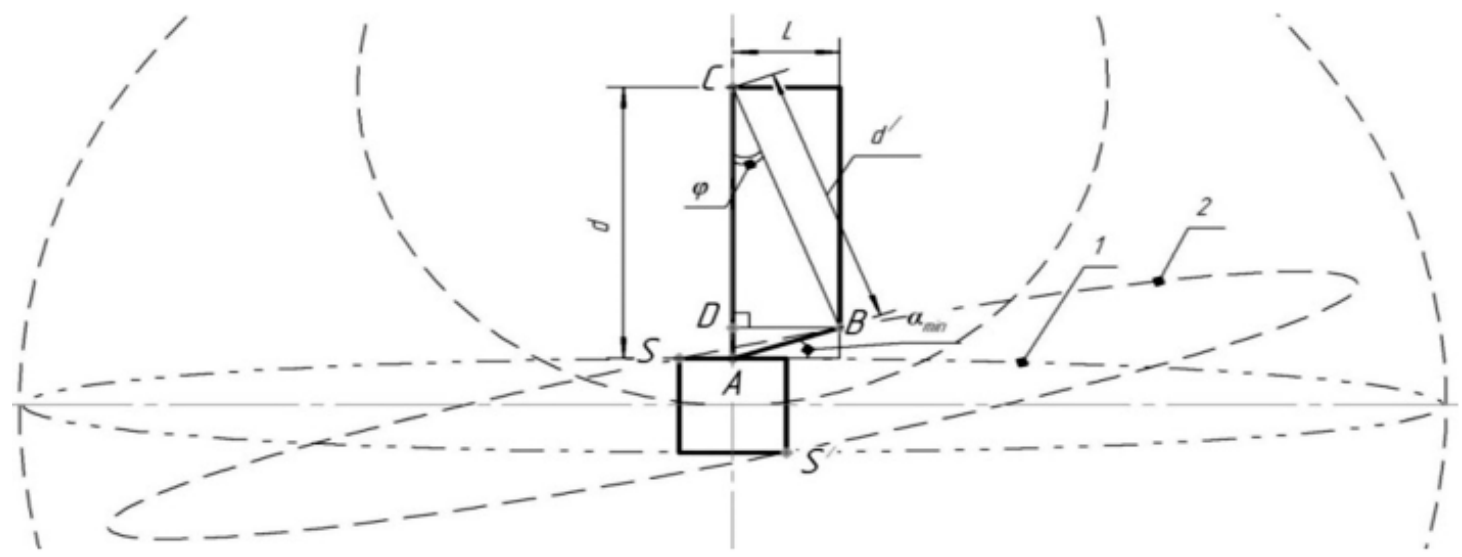

Fig. 3. Design scheme: 1 - trajectory of the apex of the cutting edge; 2 - the trajectory of the point of the rear surface; $A$ - top of cutting edge; $B$ - point of the rear surface of the instrument; $S, S$ / - rear surface of the contact point of the tool and the workpiece surface; $d$-the distance from the tool center to the top of the cutting edge; $L$ - the width of the tool body; $d /$ - distance from the center point of the tool to the rear surface; $\varphi$ - angle; $\alpha$ - the minimum clearance angle of the cutting tool

For this, consider the dependence of describing the path of the cutting edge and the apex point of the rear surface of the instrument, the trochoid 1 and 2 respectively.

The system of equations of the trochoid 1 at a ratio of the radii of the planetary gear and satellite $R / r=2$, will look like:

$$
\begin{aligned}
& x(t)=r \cdot \cos (t)+d \cdot \cos (t), \\
& y(t)=r \cdot \sin (t)-d \cdot \sin (t),
\end{aligned}
$$

where $x, y$ - the coordinates of the trochoid; $r$ - the radius of the wheel, which is located on the shaft chisel unit (mm); $d$-distance from the center to the top of the tool cutting edge (mm), $t$-parameter characterizing the rotation of the tool about its axis. Trochoid 2 is rotated relative to the trochoid 1 by an angle $\varphi$, its equation is:

$$
\begin{aligned}
& x^{\varphi}(t)=x(t) \cdot \cos (\varphi)-y(t) \cdot \sin (\varphi), \\
& y^{\varphi}(t)=x(t) \cdot \sin (\varphi)+y(t) \cdot \cos (\varphi),
\end{aligned}
$$

where $t$ - parameter characterizing the rotation of the tool about its axis, $\varphi-$ angle of rotation relative to the trochoid trochoids 21 (deg).
By substituting $x(t), y(t)$ we transform the system of equations to the form:

$$
\begin{gathered}
x^{\varphi}(t)=\left(r \cdot \cos (t)+d^{\prime} \cdot \cos (t)\right) \cdot \cos (\varphi)- \\
\quad-\left(r \cdot \sin (t)-d^{\prime} \cdot \sin (t)\right) \cdot \sin (\varphi), \\
y^{\varphi}(t)=\left(r \cdot \cos (t)+d^{\prime} \cdot \cos (t)\right) \cdot \sin (\varphi)+ \\
\quad+\left(r \cdot \sin (t)-d^{\prime} \cdot \sin (t)\right) \cdot \cos (\varphi),
\end{gathered}
$$

where $d^{\prime}-$ distance to the tool center point on the rear surface $(\mathrm{mm}), t$ - parameter characterizing the rotation of the tool about its axis, $\varphi-$ angle of rotation relative to the trochoid 1 to trochoid 2 (deg).

To determine the points of intersection of two trochoid equate equation (1), (3) in one of the coordinates, such as the $x$-coordinate.

Obtain the following equation:

$$
\begin{gathered}
\left(r \cdot \cos (t)+d^{\prime} \cdot \cos (t)\right) \cdot \cos (\varphi)- \\
-\left(r \cdot \sin (t)-d^{\prime} \cdot \sin (t)\right) \cdot \sin (\varphi)= \\
\quad=r \cdot \cos (t)+d \cdot \cos (t)
\end{gathered}
$$


Assume that $t=\pi / N$, as processed tetrahedron, where $N$ - number of facets of the polyhedron $d^{\prime}=L / \sin (\varphi) ; d^{\prime}-$ distance of the tool center to a point on the rear surface $(\mathrm{mm})$; $L$ - the width of the tool body (mm), we obtain:

$$
\begin{gathered}
\left(r+\frac{L}{\sin (\varphi)}\right) \cdot \cos \left(\frac{\pi}{2 N}\right) \cdot \cos (\varphi)- \\
-\left(r-\frac{L}{\sin (\varphi)}\right) \cdot \sin \left(\frac{\pi}{2 N}\right) \cdot \sin (\varphi)= \\
=(r+d) \cdot \cos \left(\frac{\pi}{2 N}\right)
\end{gathered}
$$

For solving the equation numerically using software package Mathcad, possible to determine the angle $\varphi$.

Having considered the right-angled triangles $\triangle B C D$ and $\triangle B D A$ we can calculate

$$
\operatorname{ctg}(\varphi)=\frac{d-L \cdot \operatorname{tg}\left(\alpha_{\min }\right)}{L}
$$

Hence, the required minimum allowable rear corner tool is:

$$
\alpha_{\min }=\operatorname{arctg}\left(\frac{d-L \cdot \operatorname{ctg}(\varphi)}{L}\right)
$$

where $d$-distance from the center to the top of the tool cutting edge $(\mathrm{mm}), L$ - width of the tool body $(\mathrm{mm}), \varphi$ - angle of rotation relative to the trochoid 1 to trochoid $2(\mathrm{deg})$.

Thus, the resulting analytical dependence rear corner allows you to assign a tool given to provide a basic requirement - the lack of contact of the back surface of the tool and the workpiece, thus providing the ability to handle sided surfaces with a variable profile of planetary by turning on automatic.

\section{Determination of the angles kinematic value}

Values of the angular kinematic parameters associated with the relative motion of the tool relative to the workpiece at each point considered cutting edge. They are calculated based on the values of the parameters, taking into account the mutual arrangement of the velocity vectors of the primary and secondary movements, as well as related planes and surfaces [6].

A characteristic feature of the method of forming the variable-sided surface profile of a change in the kinematic angles front and rear cutting edges, which depends on several parameters [7].

It should be noted that tool rake angle $\gamma$ influences the strength of the cutting wedge. With increasing rake angle facilitated cutting conditions, reduced distortion and improved surface quality. The strong increase of rake angle results in a significant weakening of the cutting edge. When processing hard and brittle metal cutters used with small rake angles and planning soft and ductile steels are trying to use a tool with a relatively large front corners. Rear tool angle $\alpha$ is used to reduce friction between the rear surface of the tool and the workpiece, which reduces the heating of the tool and result in an increase in its strength.
To assign rational geometrical parameters of the tool during the processing tool with the planetary motion is necessary to set the change in the front and rear angles during processing.

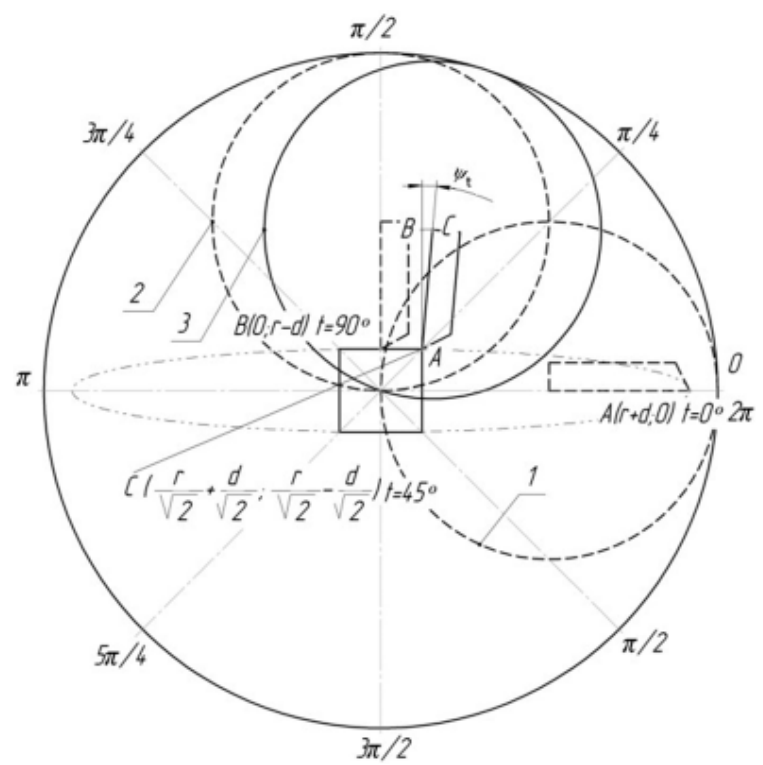

Fig. 4. Scheme vertex moving the tool cutting edge during machining

To simplify the calculation of kinematic front and rear corners, consider the process of shaping sided surfaces with variable profile at some point cut surface, which will correspond to the distance $\mathrm{d}$ from the center to the top of the tool cutting edge.

Complex motion of cutting tool consists of two simple movements - rotation around its axis of rotation relative to the workpiece. Consider the characteristic points, which will be top of the cutting tool at a time. Vertex position of the cutting edge at any given time determines the parameter $t$.

We will start the path of movement of the tool at the point $(r+d$; 0 ), where $t=0 \square$ (item 1 ), the position of the point at which the kinematic geometric angles are equal, corresponds to the coordinates $(0 ; r-d)$, where $t=90 \square$ (item 2). Consequently, the position of the top of the cutting edge of the tool insertion points corresponds to the $t=45 \square$ (key 3). Thus, for determining the change of the front and rear kinematic tool angles necessary to determine the difference in the angles of rotation of the tool at the coordinates $(0 ; r-d)$, at $t=90$ and point coordinates $\left(\frac{r}{\sqrt{2}}+\frac{d}{\sqrt{2}} ; \frac{r}{\sqrt{2}}-\frac{d}{\sqrt{2}}\right)$, at $t=45 \square$, corresponding to the moment insertion tool.

$$
\operatorname{tg}\left(\psi_{t}\right)=\frac{B C}{A B}=\frac{x(t)}{y(t)}=\frac{r+d}{r-d} \cdot \operatorname{ctg}(t)
$$

where $r$-radius of the satellite; $d$-distance from the center to the top of the tool cutting edge.

$$
\psi_{t}=\frac{\pi}{2}-\operatorname{arctg}\left(\frac{r+d}{r-d} \cdot \operatorname{ctg}\left(\frac{\pi}{N}\right)\right)
$$

where, $N$ - number of faces. Since processed tetrahedron $N=4$, the formula becomes: 


$$
\psi_{t}=\frac{\pi}{2}-\operatorname{arctg}\left(\frac{r+d}{r-d}\right)
$$

It should be noted that the amount of change of the front and rear angles are described by kinematic dependence hence the change in the rake angle value corresponds to a change of the angle adjustable kinematic.

Thus, the resulting analytical dependence to determine the kinematic change the front and rear corners in the process of shaping sided surfaces with a variable profile of planetary turning and assign them to the rational parameters taking into account technological processing modes.

\section{Experimental research}

In order to confirm the adequacy, theoretical propositions have been studied experimentally.

For the experiment purposed two experimental setups with different dimensions of planetary gear builder were made, namely with a radius of a gear wheel with internal teeth and $R_{1}=100 R_{2}=200 \mathrm{~mm}$

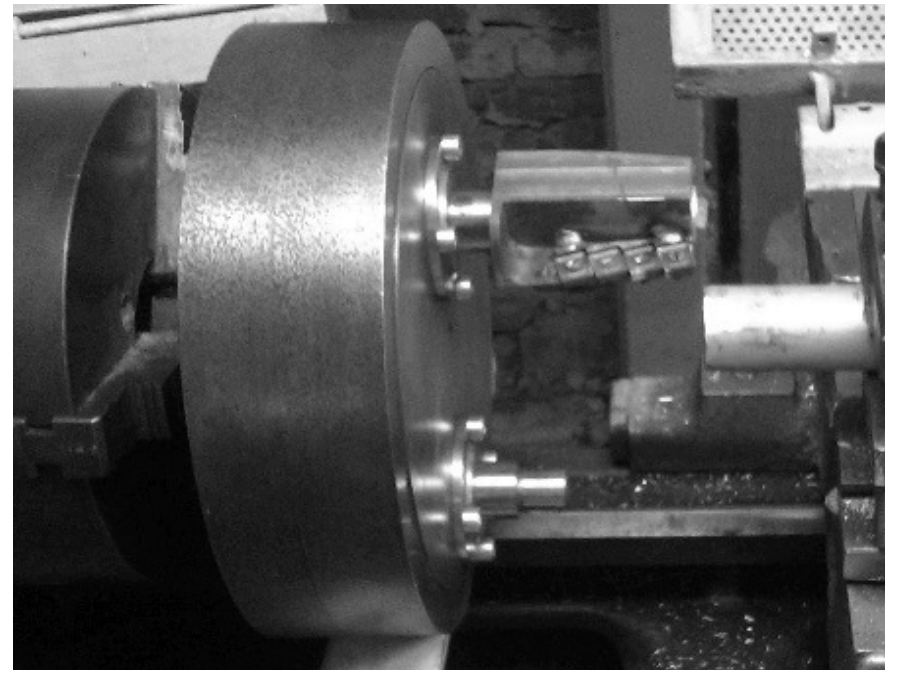

Fig. 5. Research machinery

There were also made blanks springs with different variations of the hex sizes, cutting tools, which is a unit with chisel inserts carbide with different variation of the size of their rear corner. Matrix experiment is presented in Table 1

TABLE 1

Experiment matrix

\begin{tabular}{|c|c|c|c|c|c|c|c|c|c|c|c|c|c|c|}
\hline \multirow{2}{*}{ № } & \multicolumn{2}{|c|}{$X_{1}$} & \multicolumn{2}{|c|}{$X_{2}$} & \multicolumn{2}{|c|}{$X_{3}$} & \multicolumn{2}{|c|}{$X_{4}$} & \multicolumn{3}{|c|}{ Tolerance } & \multirow{2}{*}{$\ln \Delta_{1}$} & \multirow{2}{*}{$\ln \Delta_{2}$} & \multirow{2}{*}{$\ln \Delta_{3}$} \\
\hline & code & $b$ & code & $h$ & code & $r$ & code & $\varphi$ & $\Delta_{1}$ & $\Delta_{2}$ & $\Delta_{3}$ & & & \\
\hline 1 & 2 & 3 & 4 & 5 & 6 & 7 & 8 & 9 & 10 & 11 & 12 & 13 & 14 & 15 \\
\hline 1 & - & 11 & - & 11 & - & 100 & - & 10 & 3,2 & 3,7 & 4,2 & 1,930 & 1,844 & 1,749 \\
\hline 2 & + & 24 & - & 11 & - & 100 & - & 10 & 7,1 & 7,6 & 7,6 & 1,037 & 0,884 & 0,703 \\
\hline 3 & - & 11 & + & 24 & - & 100 & - & 10 & 3,3 & 3,7 & 4,2 & 1,913 & 1,841 & 1,761 \\
\hline 4 & + & 24 & + & 24 & - & 100 & - & 10 & 7,5 & 7,8 & 8,1 & 0,948 & 0,824 & 0,678 \\
\hline 5 & - & 11 & - & 11 & + & 200 & - & 10 & 0,8 & 1,5 & 2,14 & 2,224 & 2,146 & 2,062 \\
\hline 6 & + & 24 & - & 11 & + & 200 & - & 10 & 2,9 & 3,47 & 4,0 & 1,964 & 1,876 & 1,780 \\
\hline 7 & - & 11 & + & 24 & + & 200 & - & 10 & 1 & 1,5 & 1,8 & 2,201 & 2,148 & 2,093 \\
\hline 8 & + & 24 & + & 24 & + & 200 & - & 10 & 3,2 & 4,0 & 4,1 & 1,929 & 1,869 & 1,805 \\
\hline 9 & - & 11 & - & 11 & - & 100 & + & 20 & 2,6 & 3,5 & 3,8 & 2,859 & 2,791 & 2,799 \\
\hline 10 & + & 24 & + & 24 & - & 100 & + & 20 & 7,9 & 7,4 & 7,2 & 2,496 & 2,539 & 2,580 \\
\hline 11 & - & 11 & + & 24 & - & 100 & + & 20 & 3 & 4,3 & 3,5 & 2,834 & 2,821 & 2,808 \\
\hline 12 & + & 24 & - & 11 & + & 200 & + & 20 & 3,7 & 3,8 & 3,5 & 2,791 & 2,791 & 2,806 \\
\hline 13 & - & 11 & - & 11 & + & 200 & + & 20 & 1,2 & 1,6 & 1,9 & 2,938 & 2,917 & 2,896 \\
\hline 14 & + & 24 & + & 24 & + & 200 & + & 20 & 2,4 & 2,9 & 4,0 & 2,872 & 2,827 & 2,781 \\
\hline 15 & - & 11 & + & 24 & + & 200 & + & 20 & 0,1 & 1 & 1,5 & 2,995 & 2,946 & 2,894 \\
\hline 16 & + & 24 & - & 11 & - & 100 & + & 20 & 7,2 & 7,6 & 7,2 & 2,553 & 2,519 & 2,506 \\
\hline
\end{tabular}

The results of processing raw experimental data was obtained by the empirical formula, which generalizes the influence of various factors on the variation of the kinematic front and rear corners of the cutting edges of cutting tools.

$$
\psi_{\text {exp. }}=2,51 \cdot \frac{R^{0,91} \cdot \alpha^{4,08} \cdot b^{(0,8 \ln R+0,79 \ln \alpha)}}{b^{6,63} \cdot R^{0,95 \ln \alpha}}
$$

where: $\psi_{\text {exp }}-$ kinematic modified front and rear corners of the tool; $b$ - width of the hexagonal surface of the workpiece springs, $R$-radius of the gear with internal teeth, $\alpha$-geometric rear corner.
Comparative analysis showed that the discrepancy between the experimental and theoretical results is less than 5\%, which confirms the adequacy of the mathematical relationships.

Thus, as a result of the experiment was obtained and verified by the empirical dependence of cutting corners in forming blanks sided sections of railway springs, which will take into account the change in the value of cutting corners when assigning parameters of the cutting wedge cutting tools.

To verify the results of theoretical studies to determine the variation of the kinematic front and rear angles of cutting tools has been developed an empirical relationship for determining 
the minimum value back angle of the cutting tool, obtained by multivariate experiment.

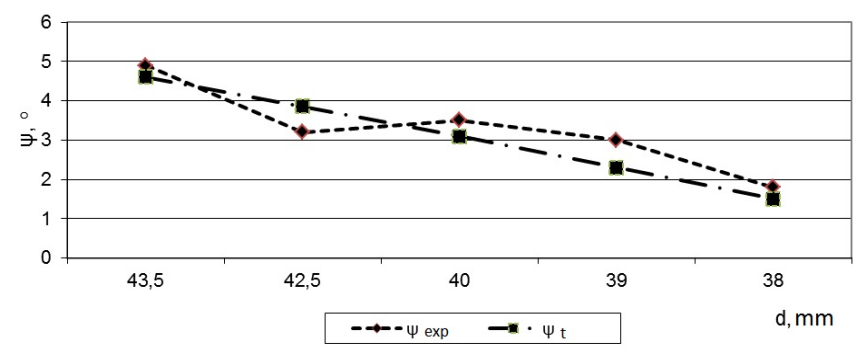

Fig. 6. Comparison of theoretical and experimental research

The experimental technique is as follows: prepared blanks of different with different sided surfaces, which in turn were mounted on a lathe cutting tool performs the role of the unit with the chisel on it cube plastic material. Preform is heated, and by turning the planetary gear unit incisive, made an imitation of the blade processing. When this chisel mounted on a cubic plastic

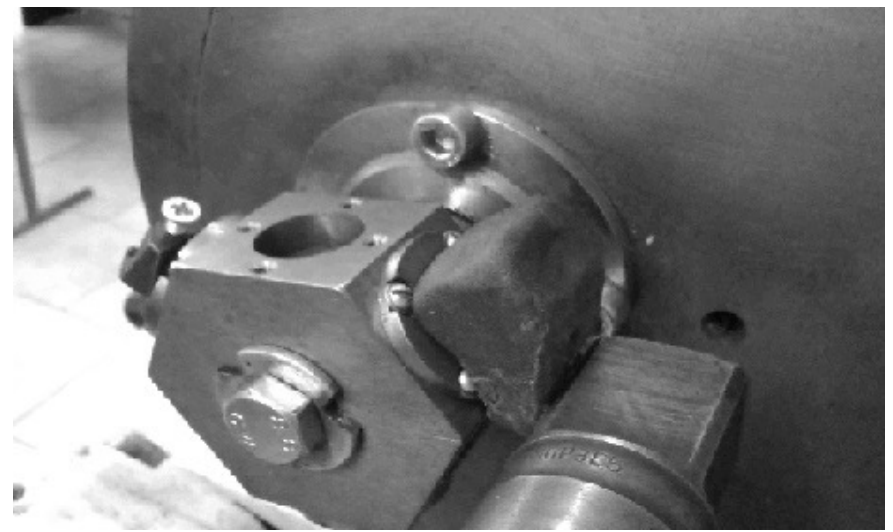

Fig. 7. Experiment fragment

block material heated preform is removed a certain layer of the material thus formed rear surface of the cutting tool made of the angle $\alpha$ measured values for the minimum profile shaping surface without encountering the cutting insert the rear surface of the workpiece or the tool holder.

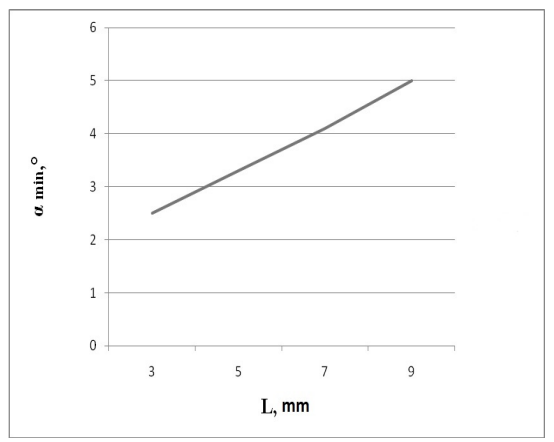

a)

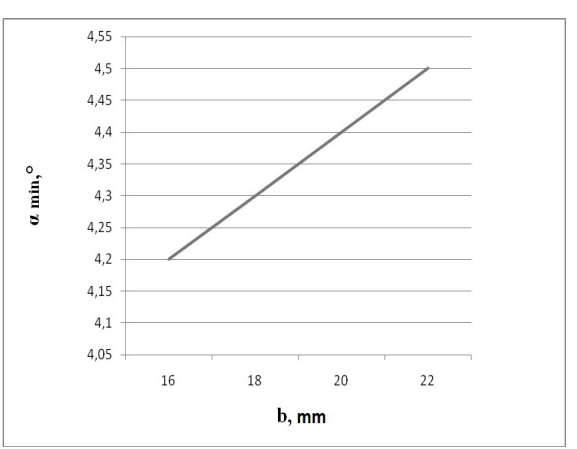

b)

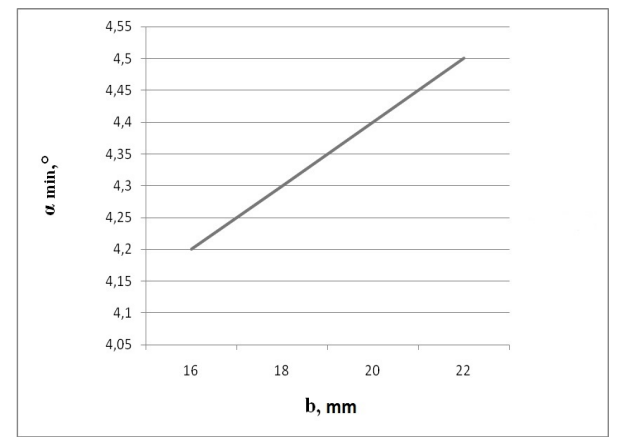

c)

Fig. 8. a) - A plot of the minimum adjustable angle $\alpha$ min cutting tool from the tool body width $L$; b) - Graph of the minimum angle $\alpha$ min adjustable cutting tool on the width $\mathrm{b}$ of the resulting profile; c) - Graph of the minimum angle $\alpha_{\min }$ adjustable cutting tool on the height h of the resulting profile

TABLE 2

Experiment matrix

\begin{tabular}{|c|c|c|c|c|c|c|c|c|c|c|c|c|c|c|}
\hline \multirow{2}{*}{ № } & \multicolumn{2}{|c|}{$X_{1}$} & \multicolumn{2}{|c|}{$X_{2}$} & \multicolumn{2}{|c|}{$X_{3}$} & \multicolumn{2}{|c|}{$X_{4}$} & \multicolumn{3}{|c|}{ Tolerance } & \multirow{2}{*}{$\ln \Delta_{1}$} & \multirow{2}{*}{$\ln \Delta_{2}$} & \multirow{2}{*}{$\ln \Delta_{3}$} \\
\hline & code & $b$ & code & $h$ & code & $R$ & code & $L$ & $\Delta_{1}$ & $\Delta_{2}$ & $\Delta_{3}$ & & & \\
\hline 1 & 2 & 3 & 4 & 5 & 6 & 7 & 8 & 9 & 10 & 11 & 12 & 13 & 14 & 15 \\
\hline 1 & - & 11 & - & 11 & - & 100 & - & 3 & 5 & 5,5 & 5 & 1,60 & 1,75 & 1,60 \\
\hline 2 & + & 24 & - & 11 & - & 100 & - & 3 & 9 & 10 & 9,5 & 2,19 & 2,30 & 2,25 \\
\hline 3 & - & 11 & + & 24 & - & 100 & - & 3 & 6 & 6,3 & 6,5 & 1,79 & 1,84 & 1,87 \\
\hline 4 & + & 24 & + & 24 & - & 100 & - & 3 & 13 & 13 & 13,5 & 2,56 & 2,56 & 2,60 \\
\hline 5 & - & 11 & - & 11 & + & 200 & - & 3 & 2,5 & 2 & 2,5 & 0,91 & 0,69 & 0,91 \\
\hline 6 & + & 24 & - & 11 & + & 200 & - & 3 & 4,9 & 5 & 4,8 & 1,58 & 1,60 & 1,56 \\
\hline 7 & - & 11 & + & 24 & + & 200 & - & 3 & 2,5 & 2,7 & 2 & 0,91 & 0,99 & 0,69 \\
\hline 8 & + & 24 & + & 24 & + & 200 & - & 3 & 5 & 5 & 5,3 & 1,60 & 1,60 & 1,66 \\
\hline 9 & - & 11 & - & 11 & - & 100 & + & 10 & 9 & 9,3 & 9,3 & 2,19 & 2,23 & 2,23 \\
\hline 10 & + & 24 & + & 24 & - & 100 & + & 10 & 18 & 17,3 & 17,5 & 2,89 & 2,85 & 2,86 \\
\hline 11 & - & 11 & + & 24 & - & 100 & + & 10 & 11 & 11 & 11,3 & 2,39 & 2,39 & 2,42 \\
\hline 12 & + & 24 & - & 11 & + & 200 & + & 10 & 6,5 & 6,5 & 6,3 & 1,87 & 1,87 & 1,84 \\
\hline 13 & - & 11 & - & 11 & + & 200 & + & 10 & 5 & 5 & 4,5 & 1,609 & 1,60 & 1,50 \\
\hline 14 & + & 24 & + & 24 & + & 200 & + & 10 & 7 & 7 & 7,2 & 1,94 & 1,94 & 1,97 \\
\hline 15 & - & 11 & + & 24 & + & 200 & + & 10 & 5,3 & 5,3 & 5,5 & 1,66 & 1,66 & 1,70 \\
\hline 16 & + & 24 & - & 11 & - & 100 & + & 10 & 14,3 & 14,5 & 14,5 & 2,66 & 2,67 & 2,67 \\
\hline
\end{tabular}


Figures 10-12 shows the dependence of the minimum value of the posterior angle of the tool on various parameters.

Graphs express the linear dependence of the variable parameter with other parameters constant. These changes may be expressed as an empirical relationship as a result of the multifactorial experiments. This will take into account and carry out the necessary corrections on the rear corner of the stage of manufacture of the instrument.

To study the dependence of the change amin-minimum rear corner on the parameters of the planetary mechanism, namely the radius of the gear with internal teeth $R$, the width and height parameters granite surface $b$ and $h$, respectively, the tool body thickness parameter $L$, and obtain empirical data was conducted multifactorial experiment

The result of processing of the experimental data was obtained by the empirical formula, which generalizes the influence of various factors on the minimum value $\alpha_{\min }$ - rear corner.

$$
\alpha_{\min }=7,1 \cdot \frac{b^{1,61} \cdot h^{0,18} \cdot L^{1,47}}{R^{1,18} \cdot b^{(0,38 \ln L)}}
$$

where $b$-width of the workpiece surface profile spring, $h$ - the height of it, $R$ - the radius of the toothed wheel with internal teeth, $L-$ the thickness of the cutting plate or the tool body.

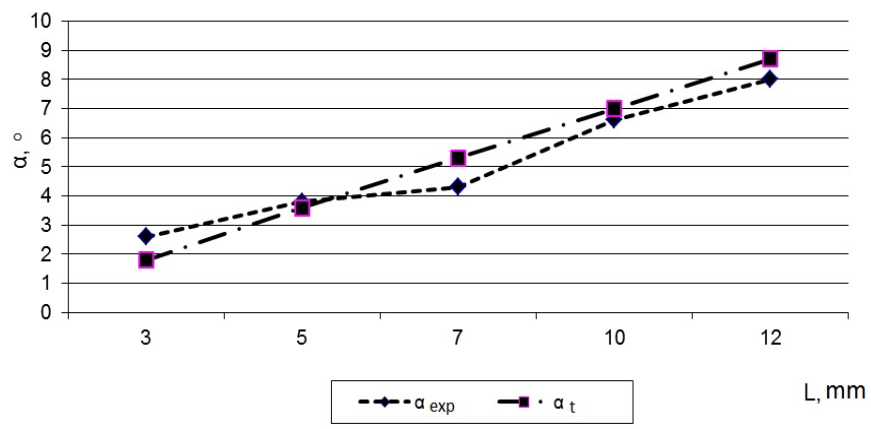

Fig. 9. Compliance with the theoretical data to the results of experimental studies

Thus investigated the effect of process parameters affecting the amount of the minimum value of the posterior angle of the cutting tool in the processing of the hexagonal blanks spring rail transport planetary accurate. Conducted multivariate experiment and received empirical model of the effect of processing parameters on the value of the minimum posterior angle of the cutting edges.

Comparative analysis showed that the discrepancy between the experimental and theoretical results is less than 5\%, which confirms the adequacy of the mathematical relationships.

\section{Conclusion}

Thus, the implementation of a method of forming sided surfaces with a variable profile of planetary treatment provides a method for approximating a straight line polyhedron gipotro- hoids as ovals, which allows you to position the cutter blades at different angles for forming a rectangular profile in the final section of the blank.

It was revealed that in the process of forming the variablesided surface profile of the planetary turning, the rear surface of the instrument under certain structural and geometric parameters can have points of contact with the workpiece surface. To ensure no contact with the rear surface of the tool surface of the workpiece obtained mathematical relationship to determine the minimum acceptable value adjustable angle, the amount of which is influenced by several factors. Among them, a planetary gear radius $R$, the distance from the center of the cutting tool to the top edge of $d$, the diameter of the workpiece, the width of the tool body $L$. valid during cutting angle of the geometrical parameters of the cutting tool, as well as the plane in which they are measured not coincide with specified during processing. To determine the actual values of the angles at each point of the surface formed by the mathematical relationship that allows you to determine the change in kinematic front and rear corners of the tool, the application of which will provide a rational purpose cutting wedge geometry parameters, namely, the front and rear corners.

The resulting mathematical relationships can be applied in the appointment of design and process parameters shaping sided surfaces with variable profile tool with planetary motion.

\section{Acknowledgements}

The work was executed with financial support of the grant of the president of the Russian Federation for the state support of young Russian scientists - candidates of sciences MK-2653.2014.8.

\section{REFERENCES}

[1] Y. Kuznetsov, A. Samoilenko, Universal Module for Machining Polygonal Surfaces on Lathes. Oborud. Instrum. Profess., Ser. Metalloobrab 5, 58-61 (2008)

[2] M. Razumov, A. Pykhtin, A. Maslennikov, Error of polyhedral profiles in single-cutter shaping by means of a planetary mechanism. Russian Engineering Research 9, 681-684 (2012).

[3] S. Emelyanov, A. Gladyshkin, M. Razumov, S Yatsun, Automatization of work preparation of profile rolls. Proceedings of the South-West State University 1, 113-116 (2012).

[4] D. Bekasov, V. Voronov, Experiments on the turning of noncircular profiles. Tekhnol. Mashinostr 5, 15-17 (2008).

[5] M. Razumov, Grechukhin, A. Pykhtin, Test calculation of preparations on rigidity in the course of the shaping of-faced surfaces with the variable profile planetary turning. Handbook. An engineering journal with appendix 4, 23-26 (2014).

[6] V. Vorona, I. Shchurov, Working kinematic front angle in turning a sinusoidal linear surface. Russian Engineering Research 5, 469472 (2012). 
[7] V. Vorona, S. Chinenov, Rational rear cutter angles for multipass turning of a complex sinusoidal contour surface. Russian Engineering Research 11, 1156-1157 (2011).

[8] A. Herberg, Modeling of self-induced vibrations that occur during the machining process of casting patterns with the use of the fuzzy-neural networks method. Archives of Metallurgy and Materials 3, 871-875 (2013).
[9] S. Wiewiorowska, Analysis of the influence of drawing process parameters on the mechanical properties of trip-structure steel wires. Archives of Metallurgy and Materials 2, 573-578 (2013).

[10] Z. Muskalski, Selected problems from the high-carbon steel wire drawing theory and technology. Archives of Metallurgy and Materials 2, 527-535 (2014). 